\title{
FROTTEMENT INTÉRIEUR A BASSE TEMPÉRATURE DANS LE FER DE HAUTE PURETÉ
}

\author{
J. San Juan, G. Fantozzi, C. Esnouf, F. Vanoni* et A. Bernalte ${ }^{* *}$ \\ Groupe d'Etudes de Métallurgie Physique et de Physique des Matériaux \\ (LA 341), I.N.S.A., Bât. 502, 69621 VilZeurbanne Cedex, France \\ ${ }^{*}$ C.E.N. Grenoble, D.R.F., 85 X, 38041 Grenoble Cedex, France \\ **Dpto Electricidad y Magnetismo U.N.E.D., Apdo 50487 Madrid, Espagne
}

Résumé - A 1'aide de mesures de frottement intérieur et de microfluage, nous avons étudie la sous-structure du pic $\alpha+\alpha$. Il apparaît trois composantes dont les paramètres d'activation ont été déterminês. Les composantes sont attribuejes aux dislocations non-vis dans les plans $\{110\}$ et $\{112\}$.

Abstract - The structure of the $\alpha+\alpha^{\prime}$ peak has been studied by internal friction and micro-creep experiments. Three components appear, and the activation parameters have been determined. These components are attributed to non-screw dislocations on $\{110\}$ and $\{112\}$ planes.

\section{I - INTRODUCTION}

L'interprétation des spectres de frottement intérieur basse température des métaux c.c. est toujours controversée (1). Par déformation à température ambiante le spectre de frottement intërieur du Fer- $\alpha$ comprend un large pic $\propto$ vers $28 \mathrm{~K}(1 \mathrm{hz})$ avec un epaulement $\alpha^{\prime}$ autour de $12 \mathrm{~K}$ qui ont èté mis en évidence par frottement intêrieur (2-5) et microdétormation (6). Le pic $\propto$ est gènéralement attribuẽ à la création thermiquement activee des doubles décrochements sur les dislocations non-vis $\left(71^{\circ}\right)(3-$ $8)$, tandis que diverses interprétations ont été proposées pour le pic $\boldsymbol{\alpha}^{\prime}$, Te plus souvent reliees au mouvement des dēcrochements gêométriques sur les disiocations vis (8-9). Néanmoins, la grande largeur du pic $\propto$ (5 ã 7 fois la 1 argeur d'un pic de Debye (2-3)) qui ne peut ètre attribuée à une distribution des longueurs des dislocations et des contraintes internes (5) indique qu'il existe probablement une sous-structure plus complexe.

\section{II - METHODE EXPERIMENTALE}

Les expériences sont réalisées sur des plaquettes $(0,5 \times 5 \times 40 \mathrm{~mm})$ de Fer de haute pureté obtenu au C.E.N.G., recuit $10 \mathrm{~h}$ à $400^{\circ} \mathrm{C}$ sous hydrogène purifiē $(10)$. Un champ magnétique axial de 200 oe est appliqué de façon à éliminer les effets du couplage magnéto-êlastique. Les êchantillons sont d'abord prédëformés de $3 \%$ en traction à température ambiante afin d'éliminer le pic $\beta_{\alpha}$ de frottement intérieur (11) et sont ensuite dêformés par torsion in situ à diffērentes tempëratures.

Les mesures de frottement intērieur sont réalisées à l'aide d'un pendule de torsion inversé équipé d'un microordinateur de table du type C.B.M. Commodore 4032 (12). La fréquence d'oscillation est de l'ordre de $3 \mathrm{hz}$.

Les mesures de microfluage sont réalisées en appliquant une contrainte statique de torsion (temps d'application $\approx 3 \mathrm{sec}$.) ; afin de minimiser le 1 ancement du pendule, la fréquence d'oscillation est réglée au maximum $(\approx 4,5 \mathrm{hz})$. La courbe de fluage est enregistrée en fonction au temps pendant $200 \mathrm{sec}$., la température étant maintenue constante à $\pm 0.1 \mathrm{~K}$. Les courbes de fluage aìnsì ob̀tenues à différentes températures ont étē lissées par une méthode d'addition exponentielles.

\section{III - RESULTATS EXPERIMENTAUX}

Fer déformé à température ambiante : La fig. 1 montre les spectres de frottement intérieur, pour diverses amplitudes de sollicitation, d'un êchantillon de fer prédéformē de $3 \%$ en traction à température ambiante et dëformé de $\pm 4 \%$ par torsion à 
$\mathrm{T}=300 \mathrm{~K}$.

Le spectre présente un pic vers $28 \mathrm{~K}$ très êlargi du côtē basse tempèrature (cet élargissement se met bien en evidence lorsque $l^{\prime}$ 'on représente le spectre en $1 / T$ ) semblable à 1 'ensemble $\alpha+\alpha$ ' observé par d'autres auteurs (2-4). La hauteur du pic augmente légèrement, et celui-ci se dëcale vers les basses températures quand on augmente 1'ampiitude de sollicitation. Il faut remarquer néanmoins, Ta présence d'un plateau au maximum du pic (surtout pour le spectre à taible ampiitude) qui suggère 1 'existence de deux composantes faiblement ecartées dans le domaine du spectre couramment attribuè à la composante $\alpha$.

La décomposition du spectre de frottement intérieur a êté rëalisée avec les hypothèses suivantes : a) les pics sont symétriques en $1 / T$, b) leur facteur d'élargissement est $\geqslant 1$. Cette dêcomposition fait apparaître trois composantes entre $10 \mathrm{~K}$ et $45 \mathrm{~K}$ et un fond continu qui dêcroît lentement en-dessous de $10 \mathrm{~K}$. Ces trois composantes dont le maximum est situe respectivement vers $28 \mathrm{~K}, 20 \mathrm{~K}$ et $\leqslant 15 \mathrm{~K}$ sont appellèes $\alpha_{1}, \alpha_{2}$ et $\alpha_{3}$. Le facteur d'éìargissement est êgai à 2 pour la composante $\alpha_{1}$ et de l'ordre de 1 pour les composantes $\alpha_{2}$ et $\alpha_{3}$.

Pour mieux préciser la sous-structure trouvēe dans l'ensemble $\alpha$, on a également réalisê des expëriences de microfiuage sur 1 'échantilion corresponaanc aux spectres de la Fig. 1, selon la méthode décrite en II. La Fig. 2 montre les courbes de fluage obtenues à dífférentes températures pour une contrainte statique appliquée de $\sigma_{\mathrm{s}}=10^{-4} \mu_{0}\left(\mu_{0}\right.$ ètant le module êlastique de cisaillement à $\left.4,2 \mathrm{~K}\right)$.

Fer déformé à y $5 \mathrm{~K}$ : Après aéformation à température ambiante, 1 'échantilion a étê déformé de $¥ 4 \%$ en torsion à $95 \mathrm{~K}$. Les spectres de frottement intérieur obtenus après dëformation et recuits à diffeerentes températures sont présentēs sur la Fig. 3 , (tous les spectres sont déterminés à une amplitude de sollicitation égaie à $5 \times 10^{-6}$ ).

Les courbes 1 et $2 \mathrm{Fig}$. 3 a montrent les spectres de frottement in térieur immëdiatement avant et après déformation à basse température. Après déformation à basse température on peut observer, mise à part une augmentation du fond continu, une chute de $l^{\prime}$ ensemble du pic $\propto$ accompagnée d'une augmentation du frottement intérieur entre $40 \mathrm{~K}$ et $60 \mathrm{~K}$, due à 7 'apparition de là composante $\boldsymbol{\beta}_{1}$ (13).

Les spectres de frottement intérieur après déformation basse température et recuit à $220 \mathrm{~K}, 245 \mathrm{~K}, 270 \mathrm{~K}$ et $30 \mathrm{u} \mathrm{K}$ sont donnēs par les courbes 1 à 4 de $1 \mathrm{a} . \mathrm{Fig}$. $3 \mathrm{~b}$ La composante $\boldsymbol{\beta}_{1}$ se décale vers les basses températures quand ja température de recuit augmente et atteint son maximum après recuit entre $220 \mathrm{~K}$ et $245 \mathrm{~K}$ (courbes 2 et 4 ), puiis disparait après recuit à $300 \mathrm{~K}$.

D'autre part, en comparant les spectres obtenus avant déformation basse température et après dëformation et recuit à $300 \mathrm{~K}$, on peut remarquer que la deformation basse température et $1 \mathrm{e}$ recuit à $300 \mathrm{~K}$ développent préférentiel lement la composante basse temperature de l'ensemble $\alpha$.

\section{IV - DISCUSSION}

A partir des courbes de microfluage de la Fig. 2 on mesure ia différence de dëformation $\Delta \mathbb{E}=\boldsymbol{E}\left(t_{2}\right)-\varepsilon\left(t_{1}\right)$ correspondant aux temps $t_{1}$ et $t_{2}$. Cette différence présente un maximum en tonction de la température pour chacun des processus de relaxation, Fig. 4. A ce maximum, le temps de reiaxation est donnë par:

$$
\zeta=\left(t_{2}-t_{1}\right) / \operatorname{Ln}\left(t_{2} / T_{1}\right)
$$

Ainsi, en faisant varier $t_{1}$ et $t_{2}$, on peut obtenir la variation de $\boldsymbol{\zeta}$ avec $T$ et à l'aide d'un diagramme d'arrhenius (Fig. 5) obtenir les paramètres d'activation du processus. Dans tous les cas, les courbes $\Delta \varepsilon=f(T)$ présentent trois composantes $\alpha_{1}, \alpha_{2}$ et $\alpha_{3}$ situées vers $17 \mathrm{~K}, 11 \mathrm{~K}$ et $8 \mathrm{~K}$ pour $\tau \simeq 100 \mathrm{sec}$. Les énergies d'activation de chacune des trois composantes sont données dans le tableau $\mathrm{I}$.

Les valeurs sont plus faibles que ceiles obtenues par frottement intérieur (2), (7-8), mais la diffërence des énergies trouvées par frottement intérieur 10,044 eV) et par microfluage $(0,018 \mathrm{eV})$ est parfaitement justifiable étant donnể que les contraintes mises en jeu dans les deux types diexpériences diffèrent de deux ordres de grandeur. 


\begin{tabular}{|l|c|c|c|}
\cline { 2 - 4 } \multicolumn{1}{c|}{} & $\alpha_{1}$ & $\alpha_{2}$ & $\alpha_{3}$ \\
\hline$E_{\mathrm{F.I.}}(\mathrm{eV})$ & 0.044 & - & - \\
\hline$E_{\text {Micro. Fluage (eV) }}$ & 0.018 & 0.011 & 0.008 \\
\hline$\sigma_{\mathrm{p}}\left(10^{-3} \mu\right)$ & 2.3 & 1.6 & 1.3 \\
\hline Syst. glissement & $(112)$ antimaclage & (112) maclage & $(110)$ \\
\hline
\end{tabular}

Tableau I

En effet, si on considère l'énergie d'activation apparente donnée par :

$$
E=E_{0}-v \cdot \sigma_{i}-v \cdot \sigma_{a}=E_{o}^{\prime}-v \cdot \sigma_{a}
$$

(avec $v=$ volume d'activation, $\sigma_{i}=$ contraintes internes, $\sigma_{a}=$ contrainte appliquée) l'énergie d'activation qu'on peut attendre en frottement intérieur est de l'ordre de $E^{\prime} 0, \sigma_{a}$ ëtant $\ll \sigma_{j}$. Par contre, dans le cas des expériences de microfluage, en prenant un volume d'activation $v \simeq 10 b^{3}(6)$ et une contrainte appliquée $\sigma_{a}=10^{-4} \mu_{0}$, on aboutit à une valeur non négligeable de v. $\sigma_{\mathrm{a}} \simeq 0,010 \mathrm{eV}$.

$D^{\prime}$ autre part, ij faut également tenir compte de $i$ 'interaction entre les décrochements donnée par (14):

$$
E=E_{0}^{1}-\left(\mu \cdot \sigma \cdot b^{6} / 2 \pi\right)^{1 / 2}
$$

Cette interaction conduit à un terme supplémentaire d'environ $0,04 \mathrm{eV}$ probablement surestimé. En considérant une valeur moyenne $(0,025 \mathrm{eV})$ du terme dû à $\sigma_{a}$ entre les deux extrêmes avec et sans interaction, on aboutit ainsi à une énergie d'activation $E^{\prime} o$ de l'ordre :

$$
E_{0}^{\prime}=E_{\text {mic. }}+E\left(\sigma_{a}\right)=0,018 \mathrm{eV}+0,025 \mathrm{eV}=0,043 \mathrm{eV}
$$

tout ã fajt compatible avec la valeur obtenue par frottement intérieur. Le même type de raisonnements peut être appl 1qué aux composantes $\alpha_{2}$ et $\alpha_{3}$.

La sous-structure du pic $\propto$ est géneralement interprétée de la manière suivante (3-9): Création thermiquement activée des doubles décrochements sur les dislocations non-vis $\left(71^{\circ}\right)$ pour la composante $\alpha$ et migration des décrochements géométriques sur les dislocations vis pour la composante $\alpha^{\prime}$.

L'hypothèse de la migration des décrochements géométriques sur les dislocations vis $n$ 'est pas confirmée par les rêsul tats expérimentaux. En effet, aucune des composantes du pic $\alpha \mathrm{n}^{2}$ est développée préfërentiellement par une déformation basse température, qui favorise pourtant la création de dislocations vis. De plus, $1^{\prime}$ application d'une contrainte statique importante devrait provoquer une diminution (ou disparition) du frottement intêrieur due à l'empiliement des décrochements géométriques. Expérimentalement, il est observé qu'une contrainte statique $n$ 'a aucun effet sur le spectre de frottement intérieur. Enfin, les trois composantes $\alpha_{1}, \alpha_{2}$ et $\alpha_{3}$ sont ioujours odservées dans les experiences de microfluage, quelles que soient les conditions de deformation et de recuit. Par conséquent, i i semble tout à tait improbaible que ia migration des décrochements géométriques puisse être responsable de I'une descomposantes observées.

Ainsi, les trois composantes du pic $\alpha$ peuvent ètre attribuées à la création thermiquement activee des doubles décrochements sur les dislocations non-vis. Deux hypothèses permettent d'expliquer la prësence des trois composantes :

1) L'existence d'une distribution des contraintes internes autour des 3 valeurs moyennes. Cette hypothèse est toutefois peu plausibie car les trois composantes apparaissent quelles que soient les conditions de déformation. 
2) L'activation de deux systèmes de glissement.

En effet, les dislocations 〈111〉 peuvent glisser soit dans le plan (110), soit dans ie pian de maclage (112), les deux systèmes étant observés macroscopiquement (15). Les contrainies de Peieris dans chacun des systèmes calculées par Yamaguchi et Vitek (16) sont données dans le tableau I. Les dislocations gl issant dans le système (111) $(110)$ (mixtes $71^{\circ}$ ) ont une contrainte de Peierls inférieure à celle des dislocations gijssant dans le système (111) (112)(coins). Ces dislocations, du fait de la dissynétrie dela barrière de Peierls dans le sens maclage ou antimaclage (15-16), présentent deux contraintes de Peierls.

Si i'on compare les valeurs des contraintes de Peierls calculées pour les trois systèmes de gi issement avec les ênergies d'activation de chacune des composantes (Tabieau I), nous pouvons observer une bonne corrélation si I'on attribue la composante $\alpha_{3}$ au système (110), la composante $\alpha_{2}$ au système (112) maclage et la composante $\alpha_{1}$ au système (112) antimaclage.

\section{REFERENCES}

(1) FANTOZZI G., RITCHIE I.G. : ICIFUAS-7, Journal de Physique C5, 42, p. 3 (1981).

(2) TAKITA K., SAKAMOTO K. : Scrip. Met. 4, p. 403 (1970).

(3) RITCHIE I.G., DUFRESNE J.F., MUSER P. : ICIFUAS-6, ed. par R. Hasiguti, University of TOKIO Press (1977) p. 701

(4) ASTIE P., PEYRADE J.P., GROH P. : ICIFUAS-7, Journal de Physique C5, 42, p. 91

(5) DUFKESNE J.F. : Thèse Universitê de Grenoble, Juin (1982)

(6) SAN JUAN J., NO L., FANTOZZI G., ESNOUF C., VANONI $F$. : ICIFUAS-7, Journal de Physique $\mathrm{C} 5,42, \mathrm{p} .43$ (1981).

(7) KRONMULLER A.. STEEB H., KONIG N. : ECIFUAS-2, II Nuovo Cimento 33B p. 205 (1976)

(8) HIVERT V., GROH P., FRANK W., RITCHIE I., MOSER P. : Phys. Stat. Sol. (a), 46,

(9) ASTIE P., PEYRADE J.P., GROH P. : Scrip. Met. 14, p. 611 (1980). p. 89 (1978).

(10) VANONI F. : Thèse Université de Grenodle (1973).

(11) SAN JUAN J., NO L., REMON A., ESNOUF C., FANTOZZI G., BERNALTE A. AnaTes de Fisica (A) 79, p. 34 (1983).

(i2) BOUVIER-VOLAILLE J.L., SAN JUAN J. : Cette conférence

(13) RITCHIE J.G., UUFREESINE J.F., MOJER P. : Phys. Stat. Sol. (a), 61, p. $591 \S 1980$ )

(14) HIRTH J.P., LOTHE J.: Theory of Dislocations, Mc Graw-Hi11 (1968).

(15) TAOKA T., TAKEUCHI S., FURUBAYASI E. : Journal of the Physical Society of Japan 19, p. 701 (1964).

(16) Yamaguchi M., VITEK V. : J. Phys. F : Metal Phys. 5, p. 11 (1975).

\section{REMERCIEMENTS}

J. San Juan remercie le Dpto de Fisica del Estado Solido de la Universidad del País Vasco pour son soutien financier. 

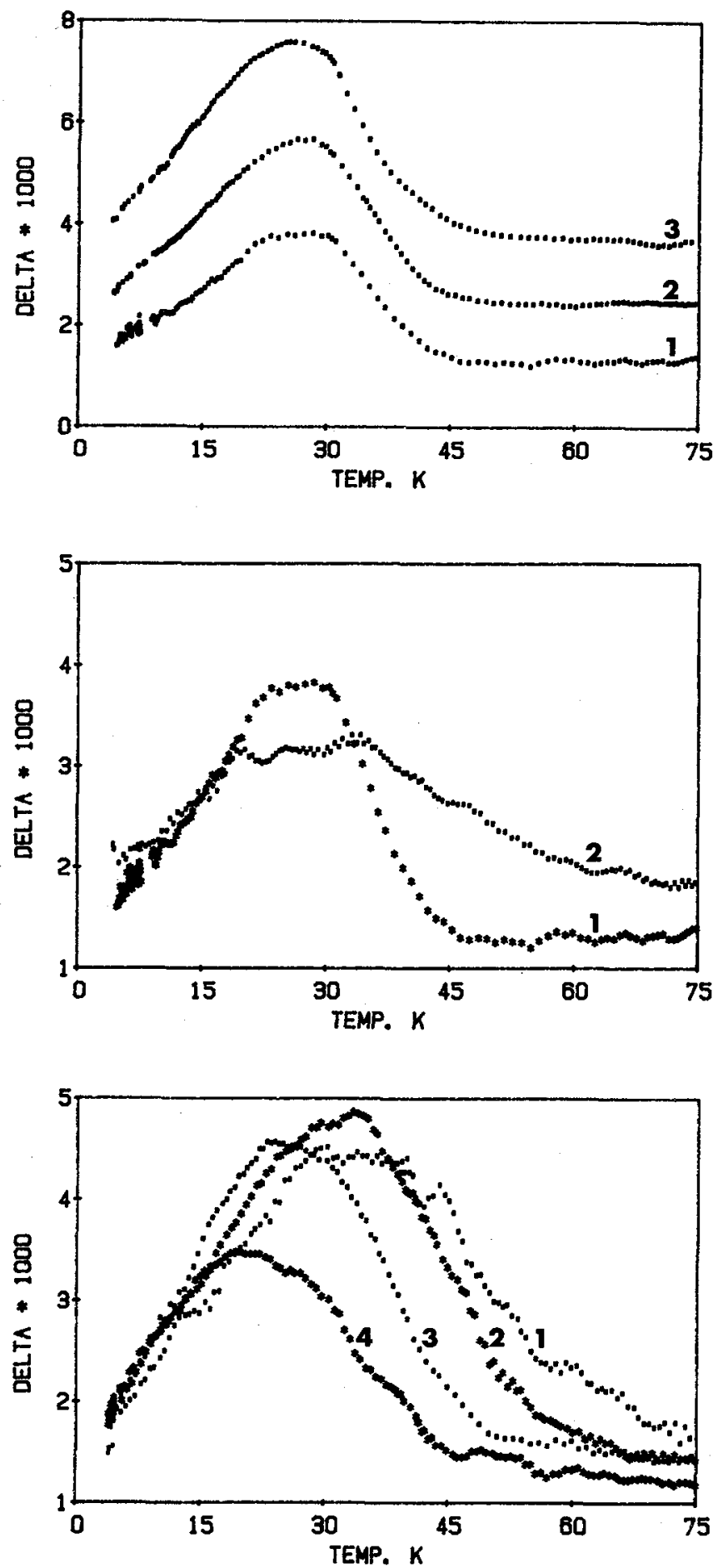

Fig.1. - Spectres de frotte ment interieur du Fer pur deformé à temperature ambian te pour differentes amplitu des de sollicitation
(1) $\varepsilon_{\mathrm{m}}=5 \times 10^{-6}$
(2) $\varepsilon_{m}=1.5 \times 10^{-5}$
(3) $\varepsilon_{m}=3.5 \times 10^{-5}$

Fig.3a.-Spectres de frotte ment inserieur du Fer pur: (1) après déformation de $+4 \%$ en torsion à temperatu $\overline{r e}$ ambiante,

(2) après une deuxième dé formition en torsion de 6 $\pm 4 \%$ à $95 \mathrm{~K}$. $\left(\varepsilon_{\mathrm{m}}=5 \times 10^{-6}\right)$

Fig. 3b.-Spectres de frotte ment interieur après defor mation basse temperature (fig.3a)et recuit à divers temperatures:
(1) $2.20 \mathrm{~K}$;
(4) $300 \mathrm{~K}$
(2) $245 \mathrm{~K}$;
(3) $270 \mathrm{~K}$ 

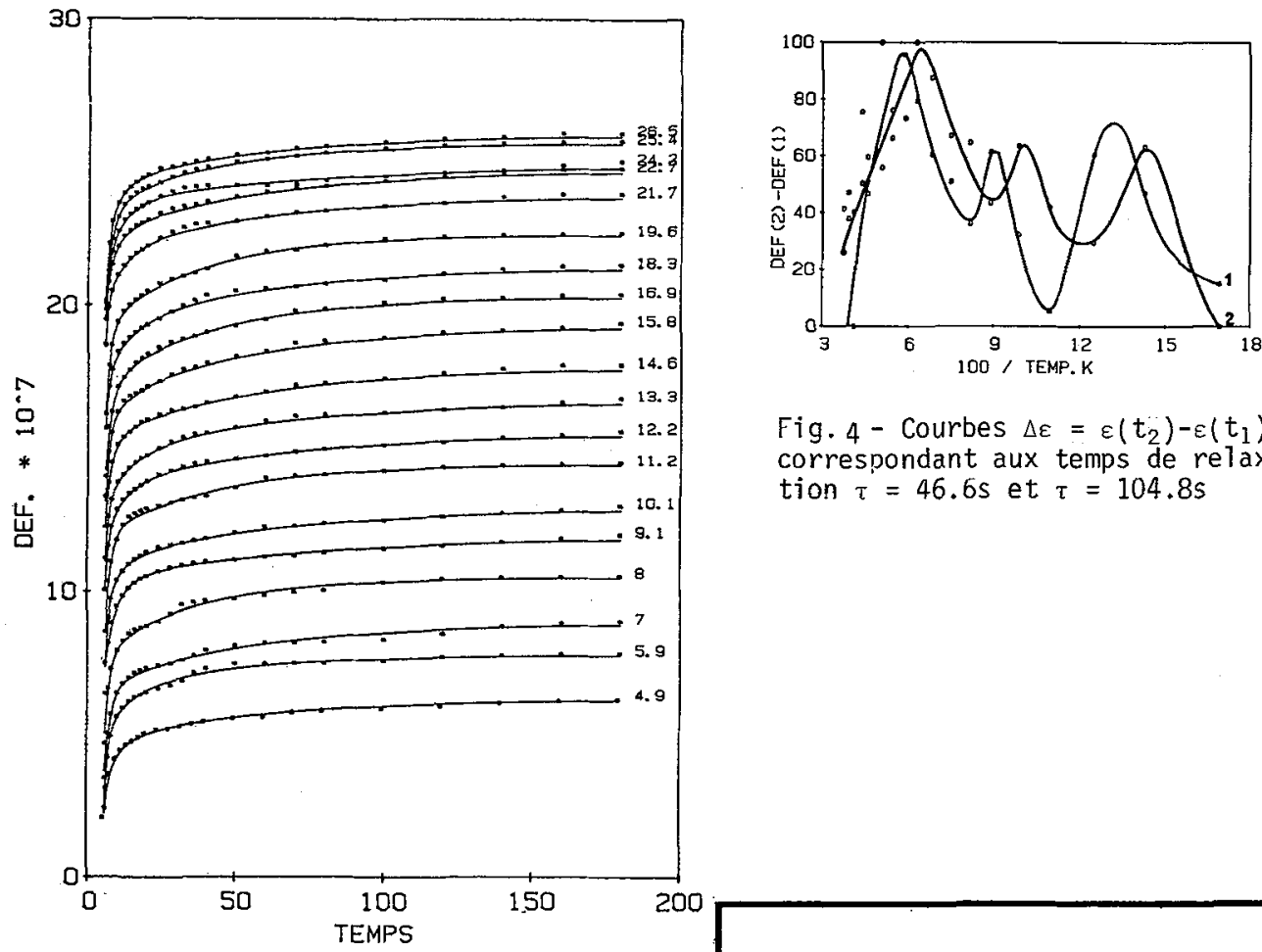

Fig. 4 - Courbes $\Delta \varepsilon=\varepsilon\left(t_{2}\right)-\varepsilon\left(t_{1}\right)$ correspondant aux temps de relaxa tion $\tau=46.6$ s et $\tau=104.8 \mathrm{~s}$

Fig. 2 - Courbes de microfluage obte nues ò differentes temperatures avec une contrainte statique égale à : $\sigma_{s}=10^{-4_{4}} \mu_{0}$

- points experimentaux - courbe lissée

Fig.5.- Diagramme d'Arrhenius pour les composantes $\alpha_{1}, \alpha_{2}$ et $\alpha_{3}$.

- Résuitats de microfluage

- Résuitats de frottement interieur

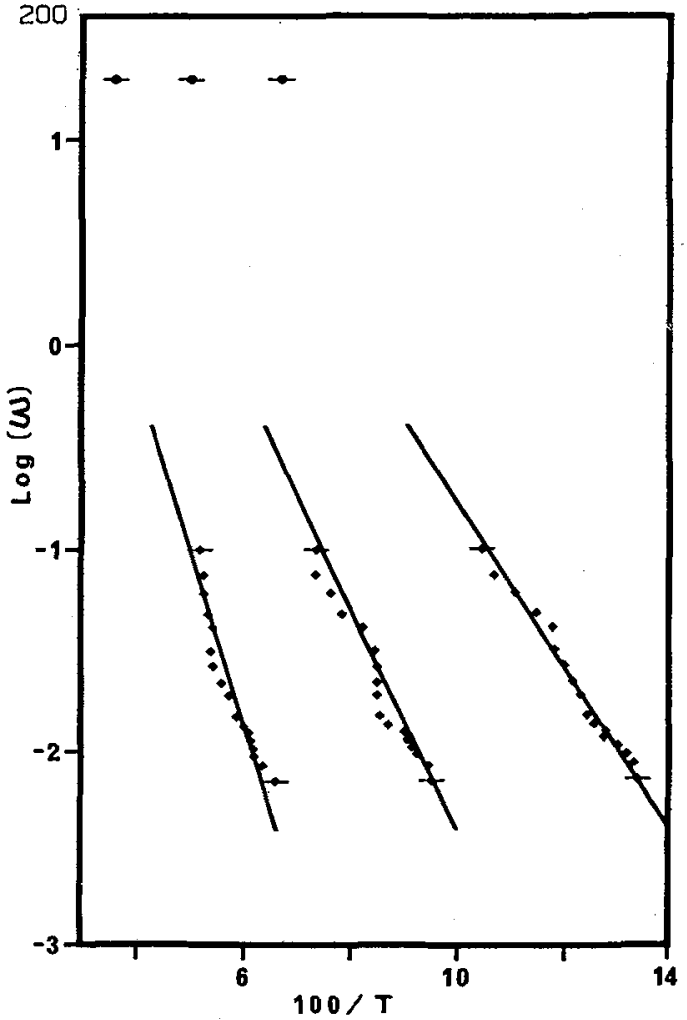

